\title{
Biomechanical evaluation of three fixation modalities for preperitoneal inguinal hernia repair: a 24-hour postoperative study in pigs
}

This article was published in the following Dove Press journal:

Medical Devices: Evidence and Research

9 December 2014

Number of times this article has been viewed

\author{
Gaëtan Guérin \\ Xavier Bourges \\ Frédéric Turquier \\ Covidien-Surgical Solutions, Research \\ and Development, Trévoux, France
}

Purpose: Tacks and sutures ensure a strong fixation of meshes, but they can be associated with pain and discomfort. Less invasive methods are now available. Three fixation modalities were compared: the ProGrip ${ }^{\mathrm{TM}}$ laparoscopic self-fixating mesh; the fibrin glue Tisseel ${ }^{\mathrm{TM}}$ with Bard ${ }^{\mathrm{TM}}$ Soft Mesh; and the SorbaFix ${ }^{\mathrm{TM}}$ absorbable fixation system with Bard ${ }^{\mathrm{TM}}$ Soft Mesh.

Materials and methods: Meshes $(6 \mathrm{~cm} \times 6 \mathrm{~cm})$ were implanted in the preperitoneal space of swine. Samples were explanted 24 hours after surgery. Centered defects were created, and samples (either ten or eleven per fixation type) were loaded in a pressure chamber. For each sample, the pressure, the mesh displacement through the defect, and the measurements of the contact area were recorded.

Results: At all pressures tested, the ProGrip ${ }^{\mathrm{TM}}$ laparoscopic self-fixating mesh both exhibited a significantly lower displacement through the defect and retained a significantly higher percentage of its initial contact area than either the Bard ${ }^{\mathrm{TM}}$ Soft Mesh with Tisseel ${ }^{\mathrm{TM}}$ system or the Bard ${ }^{\mathrm{TM}}$ Soft Mesh with SorbaFix ${ }^{\mathrm{TM}}$ absorbable fixation system. Dislocations occurred with the Bard ${ }^{\mathrm{TM}}$ Soft Mesh with Tisseel ${ }^{\mathrm{TM}}$ system and with the Bard ${ }^{\mathrm{TM}}$ Soft Mesh with SorbaFix ${ }^{\mathrm{TM}}$ absorbable fixation system at physiological pressure $(<225 \mathrm{mmHg})$. No dislocation was recorded for the ProGrip ${ }^{\mathrm{TM}}$ laparoscopic self-fixating mesh.

Conclusion: At 24 hours after implantation, the mechanical fixation of the ProGrip ${ }^{\mathrm{TM}}$ laparoscopic self-fixating mesh was found to be significantly better than the fixation of the Tisseel ${ }^{\mathrm{TM}}$ system or the SorbaFix ${ }^{\mathrm{TM}}$ absorbable fixation system.

Keywords: hernia, mesh, fixation, ProGrip ${ }^{\mathrm{TM}}$ laparoscopic self-fixating mesh

\section{Introduction}

Laparoscopic surgery is commonly used for inguinal hernia repair. Transabdominal preperitoneal and totally extraperitoneal procedures are performed with excellent results. ${ }^{1,2}$ Although there are still recurrences of hernias, their numbers have decreased considerably over the years to a rate below 3\%. ${ }^{3}$ Decreased recurrence rates might be explained by enhanced surgical techniques, the general use of meshes, and novel mesh fixation methods..$^{2-4}$ Absorbable or not, most fixation devices that are provided by health care manufacturers are tacks and sutures. This type of fixation ensures strong primary fixation of meshes, ${ }^{5}$ but can be associated with pain and discomfort. ${ }^{2,6,7}$ As recurrence rates have substantially decreased, clinicians and manufacturers now focus on improving postoperative quality of life.

The total absence of fixation can be considered in select cases. This can lead to reduced postoperative pain, ${ }^{8}$ but it might result in mesh migration. Less invasive means of fixation are now available, but it must be ensured that they provide the same fixation
Correspondence: Gaëtan Guérin Covidien-Surgical Solutions, Research and Development, II6 Avenue du Formans, 01600, Trévoux, France Tel +33474089000

Fax +33474089230

Email gaetan.guerin@covidien.com 
performance as commonly used devices. In this context, fibrin glues are increasingly used, ${ }^{9,10}$ and they exhibit an equivalent fixation strength to that of tacks on in vitro samples. ${ }^{11}$ Self-fixating meshes have been specifically designed to promote tissue integration while providing adequate fixation strength. This technology is already used in open surgery and has shown excellent clinical results. ${ }^{12}$ A mesh specifically designed for the endoscopic practice, the ProGrip ${ }^{\mathrm{TM}}$ laparoscopic self-fixating mesh (Covidien, Mansfield, MA, USA), is now available. The fixation strength of this product under physiological conditions has been evaluated and compared against other fixation modalities in this study.

Many methods are used to evaluate fixation performance. The shear force required to separate mesh from its support is generally assessed with peeling tests. ${ }^{13-15}$ This technique requires strong integration of the tested sample. Tensile tests and pull-off tests are also widely carried out..$^{5,16-19}$ In those cases, the test conditions are vastly different from those of physiological ones. Numerical models of the repaired abdominal wall could be of great interest to better understand the complexity of the mechanical response $\mathrm{e}^{20-23}$ but, as the assessment of the integration process is still challenging, studies currently focus only on the immediate postoperative time. Biomechanical test methods have been developed to characterize the mechanical behavior of hernia repair under more physiological loading conditions. With these methods, pressure chamber systems have been used to evaluate various fixation and repair techniques. ${ }^{11,24-26}$ However, only in vitro tests without implantation of meshes have been reported in the literature.

Our objective was to evaluate the fixation strength of different modalities used in inguinal hernia repair 24 hours after implantation in a pig model. Explants were placed in a pressure chamber and subjected to the equivalent of intra-abdominal pressure. Three fixation modalities were compared: the ProGrip ${ }^{\mathrm{TM}}$ laparoscopic self-fixating mesh; the fibrin glue Tisseel ${ }^{\mathrm{TM}}$ (Baxter International Inc., Deerfield, IL, USA) with Bard ${ }^{\mathrm{TM}}$ Soft Mesh (Davol, Inc., Warwick, RI, USA); and Sorbafix ${ }^{\mathrm{TM}}$ absorbable fixation system (Davol, Inc.) with BardTM Soft Mesh.

\section{Materials and methods Study design}

Eight animals were each implanted at four preperitoneal sites in the abdominal wall with three articles whose placements were randomly chosen. The three articles include the ProGrip $^{\mathrm{TM}}$ laparoscopic self-fixating mesh (number $[\mathrm{n}]=11$ ), the Bard $^{\text {TM }}$ Soft Mesh with TisseelTM $(\mathrm{n}=11)$, and the Bard ${ }^{\text {TM }}$ Soft
Mesh with SorbaFix ${ }^{\mathrm{TM}}$ absorbable fixation system $(\mathrm{n}=10)$. Mesh-containing abdominal wall samples were removed 24 hours after surgery for the analysis of mesh fixation.

\section{Materials}

The ProGrip ${ }^{\mathrm{TM}}$ laparoscopic self-fixating mesh (density before absorption, $82 \mathrm{~g} / \mathrm{m}^{2}$; density after absorption, $49 \mathrm{~g} /$ $\mathrm{m}^{2}$ ), the Bard ${ }^{\mathrm{TM}}$ Soft Mesh (density, $44 \mathrm{~g} / \mathrm{m}^{2}$ ), Tisseel ${ }^{\mathrm{TM}}$ fibrin sealant, and the SorbaFix ${ }^{\mathrm{TM}}$ absorbable fixation system (henceforth referred to as "SorbaFix"TM" or "SorbaFix"TM tacks") were used for implantation. Prolene ${ }^{\circledR}$ 5-0 stitches (Ethicon, Inc.; Johnson \& Johnson, Somerville, NJ, USA), sutures for closing subcutaneous layers (Vicryl ${ }^{\circledR} 3-0$; Ethicon, Inc.), and sutures for closing muscle layers (PDS ${ }^{\circledR}$; Ethicon, Inc.; Johnson \& Johnson) were used during the surgical procedure.

\section{Animal model}

Healthy, female swine (Sus scrofa domesticus; $\mathrm{n}=8$ ), each with a body weight between $46 \mathrm{~kg}$ and $51 \mathrm{~kg}$ at the study onset, were obtained from Gaec du Perrat in Chaleins, France. Animals were acclimated for at least 5 days in an accredited North American Science Associates, Inc. (NAMSA) facility prior to the surgical procedure. The European Directive EEC/86/609 guidelines for the care and use of laboratory animals have been observed, and the protocol was approved by the NAMSA ethical committee. Animals received standard veterinary care.

\section{Surgical procedure}

Animals were not allowed to eat overnight before the surgical procedure. Immediately prior to surgery, animals were weighed and given an intramuscular injection of atropine (atropinum sulfuricum; Aguettant, Lyon, France). Anesthesia was induced by an intramuscular injection of tiletaminezolazepam (Zoletil ${ }^{\circledR}$ 100; Virbac Corporation, Amherst, NH, USA) followed by an intravenous injection of thiopental sodium (Nesdonal ${ }^{\circledR}$; Merial, Lyon, France), and maintained by inhalation of $\mathrm{O}_{2}$-isoflurane $\left(1 \%-4 \%\right.$ mixture; Aerrane ${ }^{\circledR}$; Baxter International, Inc.). The animals were then given an analgesic treatment of buprenorphine (Buprecare ${ }^{\circledR}$; Axience, Pantin, France) and flunixin (Meflosyl ${ }^{\circledR}$; Pfizer, Inc., New York, NY, USA) by intramuscular injection, and a preventative antibiotic (amoxicillin, Duphamox LA ${ }^{\circledR}$; Pfizer, Inc.) was administered. Hair was removed from the surgical site. Skin around the abdomen was scrubbed with povidone iodine (Vetedine ${ }^{\circledR}$; Vetoquinol SA, Lure, France), wiped with $70 \%$ isopropyl alcohol, painted with povidone iodine 
solution (Vetedine ${ }^{\circledR}$ solution; Vetoquinol SA), and draped. Surgery was performed by an experienced veterinary surgeon following standard aseptic procedures. A full midline laparotomy that left the peritoneum intact was performed to access the retroperitoneal space. The lateral retroperitoneal space was bluntly dissected on both sides; the incision was made distally up to the inguinal region, proximally up to the diaphragm, and laterally to the lumbar area. Peritoneal tears were sutured with resorbable sutures $\left(\right.$ Vicryl $\left.^{\circledR} 3-0\right)$ prior to mesh implantation.

The meshes were cut into $6 \mathrm{~cm} \times 6 \mathrm{~cm}$ sections. The ProGrip ${ }^{\mathrm{TM}}$ laparoscopic self-fixating mesh was soaked in $0.9 \% \mathrm{NaCl}$ for a few seconds before implantation. The Tisseel ${ }^{\mathrm{TM}}$ fibrin sealant was defrosted at $35^{\circ} \mathrm{C}$ and prepared according to the manufacturer's instructions. The ProGrip ${ }^{\mathrm{TM}}$ laparoscopic self-fixating mesh was placed with the grips against the abdominal wall (rectus), and four stitches (Prolene $\left.{ }^{\circledR} 5-0\right)$, one at each corner of the mesh, were added to secure the mesh to the rectus muscles as a precaution against mesh dislocation. The Bard ${ }^{\mathrm{TM}}$ Soft Mesh was fixed with Tisseel $^{\mathrm{TM}}$ fibrin sealant (applied on the full surface of the side placed against the abdominal wall, according to manufacturer's instructions), and contact with the peritoneum was avoided for 5 minutes to ensure that the mesh was fixed to the rectus muscles but not to the peritoneum. Four stitches, one at each corner of the mesh, were then added, just as for the ProGrip ${ }^{\mathrm{TM}}$ laparoscopic self-fixating mesh group. For the application of the Bard ${ }^{\mathrm{TM}}$ Soft Mesh fixed with four absorbable SorbaFix ${ }^{\mathrm{TM}}$ tacks, one tack was placed at each corner of the mesh. In all animals, the two sites on each side were spaced such that at least $2 \mathrm{~cm}$ of biological tissue remained around each article. After implantation, the linea alba was closed with individual resorbable sutures $\left(\mathrm{PDS}^{\circledR}\right)$, and the subcutaneous layers were closed with continuous resorbable sutures (Vicryl $\left.{ }^{\circledR} 3-0\right)$. The skin was closed with surgical clips. The animals were allowed to recover from anesthesia in the operating room, then transported to their cages and kept under close observation. At the end of the surgery day, an analgesic treatment of buprenorphine (Buprecare ${ }^{\circledR}$ ) was administered by intramuscular injection. The position of each animal was regularly changed.

\section{Sample recovery and preparation}

Twenty-four hours after implantation, each animal was anesthetized by an intramuscular injection of tiletaminezolazepam (Zoletil $\left.{ }^{\circledR} 100\right)$ and euthanized by a lethal injection of pentobarbital solution (Dolethal ${ }^{\circledR}$; Vetoquinol SA). The abdomen was opened, and an area of tissue that contained the implant and that included at least $1 \mathrm{~cm}$ of tissue around each side of the article was excised. After explantation, a $3 \mathrm{~cm}$ defect was created in the abdominal wall (in the center of the article). To mimic a hernia, the rectus sheath tissue within a circular guide was excised using a scalpel; this method left the peritoneum intact. Sutures were then removed from the corners of the ProGrip ${ }^{\mathrm{TM}}$ laparoscopic self-fixating mesh sample and the Bard ${ }^{\mathrm{TM}}$ Soft Mesh with Tisseel $^{\mathrm{TM}}$ sample. The peritoneum was removed with no damage; hence, the samples could be visualized and could be kept for use during biomechanical testing. Preperitoneal hematomas were removed. All samples were maintained at room temperature until biomechanical testing.

\section{Biomechanical testing}

Mesh fixation was assessed using biomechanical testing adopted from Schwab et al. ${ }^{11,26}$ Sample preparation is shown in Figure 1A and B, and the biomechanical testing system containing the sample is shown in Figure $1 \mathrm{C}$. The system consists of a pressure chamber that simulates the abdominal wall cavity, a high precision pressure regulator, a pressure sensor, a displacement sensor, and a nondeformable solid support with a $3 \mathrm{~cm}$ diameter hole to support the sample. Data were recorded by a video camera and data acquisition system. The layer containing the mesh and consisting of muscle and aponeurosis tissue was used for analysis. A biological sample of at least $8 \mathrm{~cm} \times 8 \mathrm{~cm}$ was placed on a solid support. For the sole purpose of preventing air leaks, the peritoneum was repositioned on the sample; the repositioning was not considered to affect the mechanical strength of mesh fixation. Nails were placed at the edges of the sample to ensure its fixation on the support. The pressure chamber was closed, and the displacement sensor was placed in contact with the mesh. Fixation was evaluated by mesh displacement through the defect and by the assessment of the contact area of the mesh across the abdominal wall. The displacement was measured by a sensor placed below the sample. The contact area was assessed by qualitative visual evaluation and two-dimensional digital optics, and relative values were determined using ImageJ software v1.44p (National Institutes of Health, Bethesda, MD, USA). Measurements were continuously recorded with applied pressure ranging from $0 \mathrm{mmHg}$ to $450 \mathrm{mmHg}$ and were analyzed at intervals of $45 \mathrm{mmHg}$.

\section{Statistical analysis}

Mesh displacement data and mesh contact area data were statistically analyzed at two endpoints, $225 \mathrm{mmHg}$ (maximum intra-abdominal pressure in humans ${ }^{27}$ ) and $450 \mathrm{mmHg}$, by 

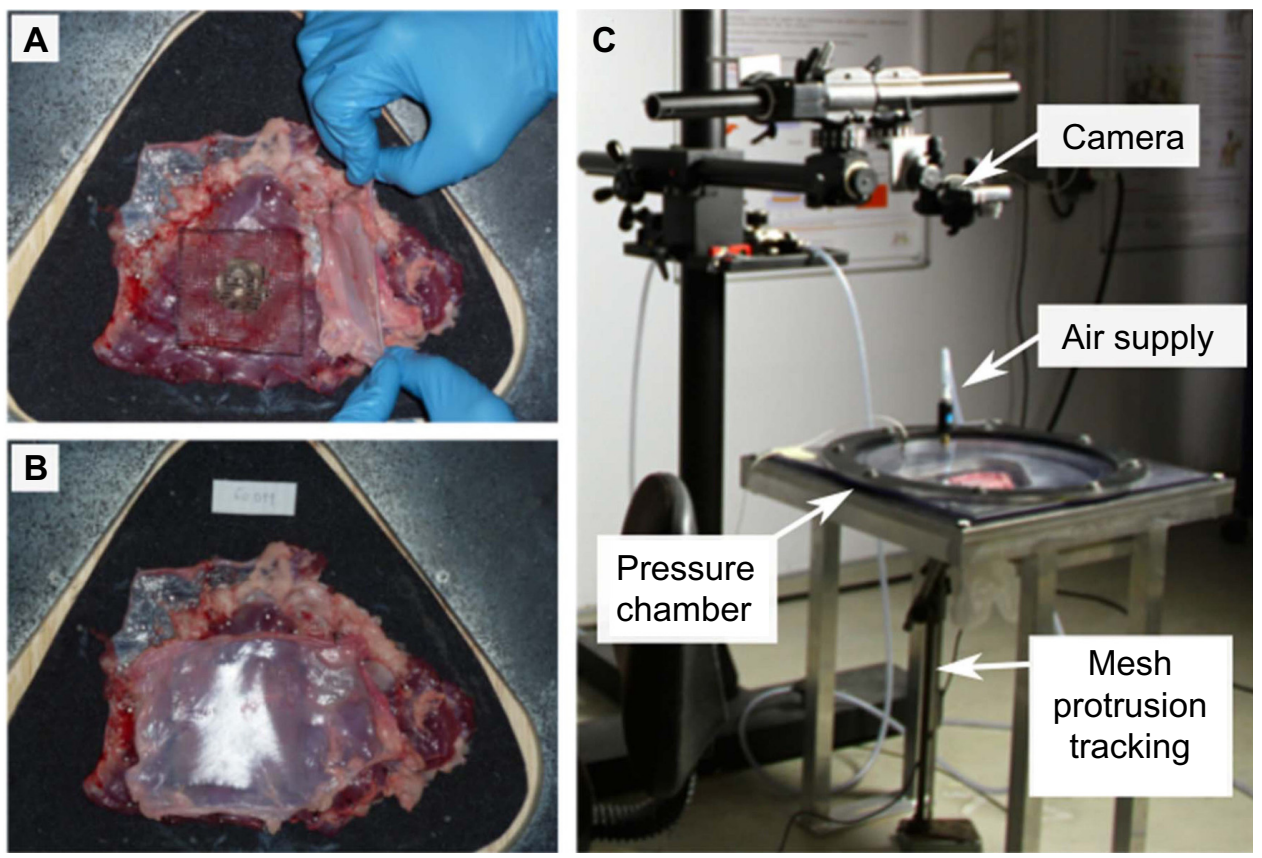

Figure I Biomechanical testing.

Notes: The peritoneum was removed from each sample to visualize the tissue that surrounded the mesh; the peritoneum was then repositioned prior to biomechanical testing. The biological sample after removal of the peritoneum and creation of the $3 \mathrm{~cm}$ defect with nails fixing the edges of the sample to the solid support (A). Biological sample with the peritoneum repositioned but not fixed to the mesh (B). Biological sample placed within the pressure chamber of the biomechanical testing system (C).

the Mann-Whitney nonparametric test. Dislocated samples were not included in the statistical analysis. Mesh dislocation data were statistically analyzed by the Fisher's exact test. Significance was determined at $P<0.05$.

\section{Results}

\section{Sample collection}

The animals were prepared for surgery, and implantation of the test and control articles was performed as described in the "Materials and methods" section. All eight animals survived the surgery, and the implant-containing biological samples from all four retroperitoneal sites in each animal were analyzed. At termination, no gross changes were observed in the tissue around the fixation site in any animal.

\section{Mesh displacement}

Mesh displacement of the three articles (ProGrip ${ }^{\mathrm{TM}}$ laparoscopic self-fixating mesh, T [test article]; Bard ${ }^{\text {TM }}$ Soft Mesh with Tisseel ${ }^{\mathrm{TM}}, \mathrm{C} 1$ [control article 1]; and Bard $^{\mathrm{TM}}$ Soft Mesh with SorbaFix ${ }^{\mathrm{TM}}, \mathrm{C} 2$ [control article 2]) was determined with increasing amounts of applied pressure. Pressure was continuously applied from $0 \mathrm{mmHg}$ to $450 \mathrm{mmHg}$, and mesh displacement is shown from $45 \mathrm{mmHg}$ to $450 \mathrm{mmHg}$ at intervals of $45 \mathrm{mmHg}$ (Figure 2). Some samples exhibited mesh dislocation; these were excluded from the analysis because no data could be recorded. At all pressures tested, ProGrip ${ }^{\mathrm{TM}}$ laparoscopic self-fixating mesh demonstrated the least displacement of the three articles. Statistical analysis was performed at $225 \mathrm{mmHg}$ and $450 \mathrm{mmHg}$. At a pressure of $225 \mathrm{mmHg}$, the ProGrip ${ }^{\mathrm{TM}}$ laparoscopic self-fixating mesh exhibited $15 \%$ less displacement than the Bard ${ }^{\mathrm{TM}}$ Soft Mesh with Tisseel $^{\mathrm{TM}}(P=0.042)$, and $23 \%$ less displacement than the Bard $^{\text {TM }}$ Soft Mesh with SorbaFix ${ }^{\mathrm{TM}}(P=0.004)$. A similar result was observed at a pressure of $450 \mathrm{mmHg}$; the displacement of the ProGrip ${ }^{\mathrm{TM}}$ laparoscopic self-fixating mesh through the defect was $17 \%$ less than that of the Bard'M

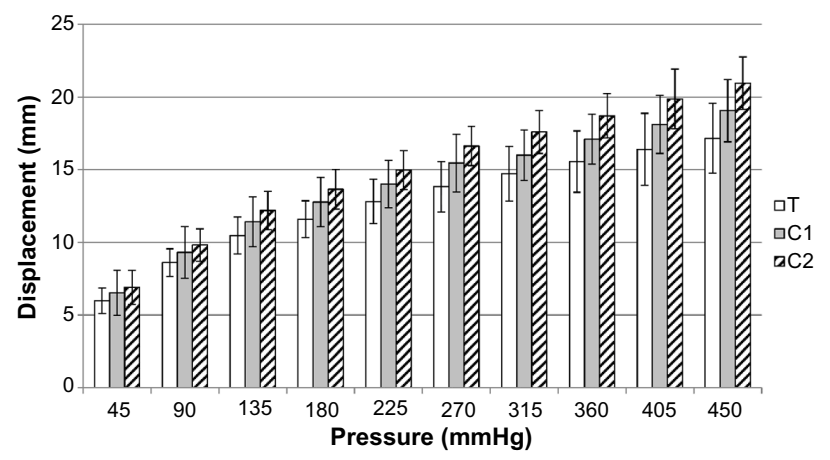

Figure 2 Mesh displacement.

Notes: Pressure was applied to the defect-containing tissue, and displacement was measured by a displacement sensor placed below the sample. Data represent the mean distance of mesh displacement through the defect, and error bars represent the standard deviation. $T$ (test article), ProGrip ${ }^{\mathrm{TM}}$ laparoscopic self-fixating mesh; $\mathrm{Cl}$ (control article I), Bard ${ }^{\mathrm{TM}}$ Soft Mesh with Tisseel ${ }^{\mathrm{TM}}$; $\mathrm{C2}$ (control article 2), Bard $^{\mathrm{TM}}$ Soft Mesh with SorbaFix ${ }^{\mathrm{TM}}$. 
Soft Mesh with Tisseel ${ }^{\mathrm{TM}}(P=0.032)$, and $25 \%$ less than the displacement of the Bard ${ }^{\mathrm{TM}}$ Soft Mesh with SorbaFix ${ }^{\mathrm{TM}}$ $(P=0.005)$.

\section{Mesh contact area}

For each sample, the contact area of the mesh with the tissue was analyzed from $45 \mathrm{mmHg}$ to $450 \mathrm{mmHg}$ at intervals of $45 \mathrm{mmHg}$. No data were recorded for samples with dislocated mesh. The ratio of the mesh contact area to the initial mesh contact area was calculated for all three types of mesh treatments for each pressure reading. The ProGrip ${ }^{\mathrm{TM}}$ laparoscopic self-fixating mesh exhibited the highest ratio of the three articles at all pressures tested (Figure 3). At $225 \mathrm{mmHg}$, the ratio for samples with ProGrip ${ }^{\mathrm{TM}}$ laparoscopic self-fixating mesh was $9 \%$ greater than that for the Bard ${ }^{\mathrm{TM}}$ Soft Mesh with Tisseel ${ }^{\mathrm{TM}}$ samples $(P=0.006)$, and $16 \%$ greater than the ratio for the Bard ${ }^{\mathrm{TM}}$ Soft Mesh with SorbaFix ${ }^{\mathrm{TM}}$ samples $(P<0.001)$. Increasing the applied pressure to $450 \mathrm{mmHg}$ led to a ratio for the ProGrip ${ }^{\mathrm{TM}}$ laparoscopic self-fixating mesh that was $21 \%$ larger than that for the Bard ${ }^{\mathrm{TM}}$ Soft Mesh with Tisseel $^{\mathrm{TM}}(P<0.001)$ and $30 \%$ larger than that for the Bard ${ }^{\mathrm{TM}}$ Soft Mesh with SorbaFix ${ }^{\mathrm{TM}}(P=0.001)$. After applying 450 $\mathrm{mmHg}$ of pressure on the biological sample, the pressure was returned to $0 \mathrm{mmHg}$, and the mesh contact area was measured again to compare the ability of the various articles to return to their initial positions. The ratios of the final mesh contact areas compared to the initial mesh contact areas are shown in Figure 4. While no significant difference was observed between the ProGrip ${ }^{\mathrm{TM}}$ laparoscopic self-fixating mesh and the Bard ${ }^{\mathrm{TM}}$ Soft Mesh with Tisseel ${ }^{\mathrm{TM}}$, a significantly higher ratio of final mesh contact area to the initial mesh contact area was observed in the ProGrip ${ }^{\mathrm{TM}}$ laparoscopic self-fixating mesh samples compared to the Bard ${ }^{\mathrm{TM}}$ Soft Mesh with SorbaFix $^{\mathrm{TM}}$ samples $(P<0.001)$.

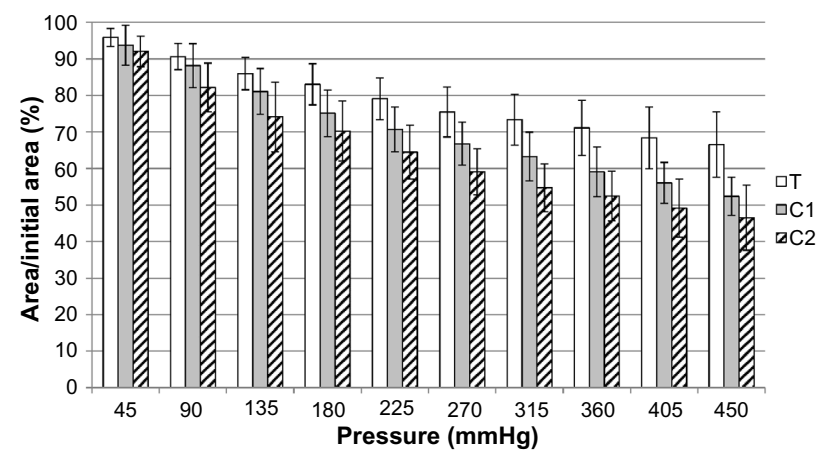

Figure 3 Mesh contact area.

Notes: Pressure was applied to the defect-containing tissue, and the contact area was measured. Data are shown as the mean percentage of mesh contact area relative to the initial mesh contact area, and error bars represent the standard deviation. $T$ (test article), ProGrip ${ }^{\mathrm{TM}}$ laparoscopic self-fixating mesh; $\mathrm{Cl}$ (control article I), Bard ${ }^{\mathrm{TM}}$ Soft Mesh with Tisseel ${ }^{\mathrm{TM}}$; C2 (control article 2), Bard ${ }^{\mathrm{TM}}$ Soft Mesh with SorbaFix ${ }^{\mathrm{TM}}$.

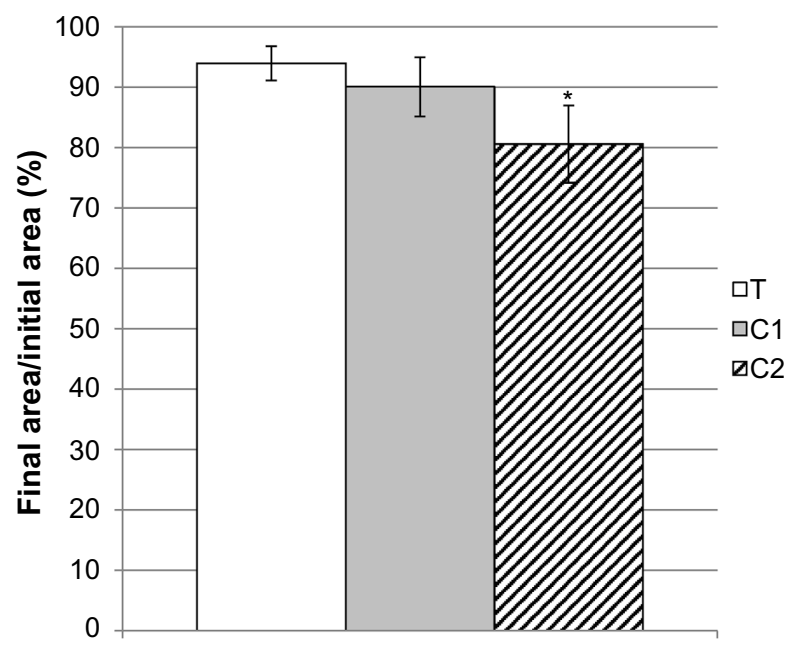

Figure 4 Ratio of final mesh surface area to initial mesh surface area. Notes: After the maximum pressure was applied to the defect-containing tissue, the pressure was returned to $0 \mathrm{mmHg}$, and the mesh contact area was measured. Data are shown as the percentage of mesh contact area relative to the initial mesh contact area. Error bars indicate the standard deviation. *Statistically significant at $P<0.001$ by the Mann-Whitney test, compared to ProGrip ${ }^{\mathrm{TM}}$ laparoscopic self-fixating mesh; T (test article), ProGrip ${ }^{\mathrm{TM}}$ laparoscopic self-fixating mesh; $\mathrm{Cl}$ (control article I), Bard ${ }^{\mathrm{TM}}$ Soft Mesh with Tisseel ${ }^{\mathrm{TM}}$; $\mathrm{C} 2$ (control article 2), Bard ${ }^{\mathrm{TM}}$ Soft Mesh with SorbaFix ${ }^{\mathrm{TM}}$.

\section{Mesh dislocation}

Mesh dislocation is a major cause of hernia recurrence, but the above measurements do not take samples exhibiting mesh dislocation into consideration. To include mesh dislocation in the overall comparison of mesh fixation, the dislocation rate of each article was recorded. Bard ${ }^{\text {TM }}$ Soft Mesh with Tisseel $^{\mathrm{TM}}$ and Bard ${ }^{\mathrm{TM}}$ Soft Mesh with SorbaFix ${ }^{\mathrm{TM}}$ were found to dislocate at physiological intra-abdominal pressure $(<225 \mathrm{mmHg})$ in some cases, and greater rates of dislocation were observed for each with increased pressure (Figure 5). No dislocation was observed for the ProGrip ${ }^{\mathrm{TM}}$ laparoscopic self-fixating mesh even at an applied pressure of $450 \mathrm{mmHg}$,

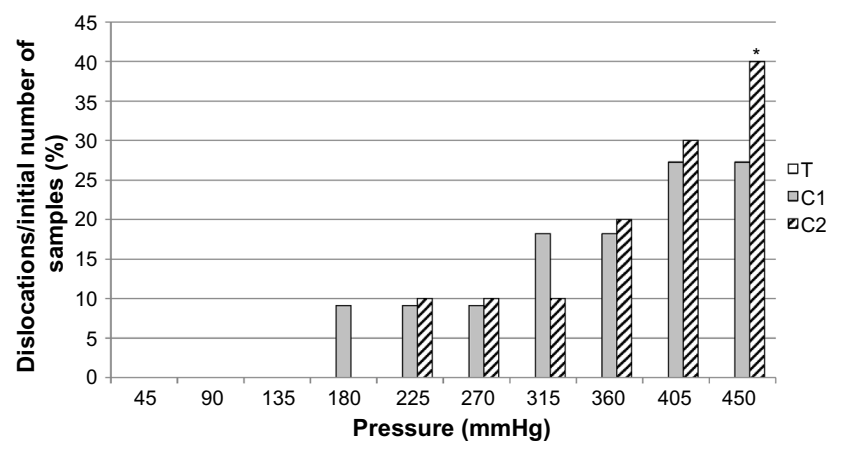

Figure 5 Mesh dislocation.

Notes: Pressure was applied to the defect-containing tissue, and mesh either remained fixed to the tissue or became dislocated. Data represent the percentage of samples with dislocated mesh. No ProGrip ${ }^{\mathrm{TM}}$ laparoscopic self-fixating mesh samples dislocated at any pressure that was tested. *Statistically significant at $P=0.035$ by Fisher's exact test, as compared to the ProGrip ${ }^{\mathrm{TM}}$ laparoscopic self-fixating mesh; $\mathrm{T}$ (test article), ProGrip ${ }^{\mathrm{TM}}$ laparoscopic self-fixating mesh; $\mathrm{Cl}$ (control article I), Bard ${ }^{\mathrm{TM}}$ Soft Mesh with Tisseel ${ }^{\mathrm{TM}}$; C2 (control article 2), Bard ${ }^{\mathrm{TM}}$ Soft Mesh with SorbaFix ${ }^{\mathrm{TM}}$. 
the maximum pressure tested. The difference in the mesh dislocation rates at $450 \mathrm{mmHg}$ between the ProGrip ${ }^{\mathrm{TM}}$ laparoscopic self-fixating mesh and the Bard ${ }^{\mathrm{TM}}$ Soft Mesh with SorbaFix $^{\mathrm{TM}}$ was significant $(P=0.035)$.

\section{Discussion}

The utilization of synthetic or biological mesh products in hernia repair provides a mechanism for strengthening defective tissue; however, hernia recurrence remains a complication for many patients. ${ }^{4,28,29}$ Innovative products have been developed in an attempt to reduce recurrence rates and improve postoperative quality of life. A comprehensive understanding of these products in regard to their ease of use, overall patient satisfaction, and long-term success will influence both their adoption in the operating room and future developments in hernia repair.

We adopted a testing device that focuses on mesh fixation to investigate different fixation modalities under physiological conditions. This setup allows for fine control of applied pressure and data measurement through the placement of sensors beneath the support and a camera above the sample. With this method of biomechanical testing, we can perform ex vivo mesh fixation studies of biological samples under intra-abdominal pressure. In this study, we compared the fixation of the ProGrip ${ }^{\text {TM }}$ laparoscopic self-fixating mesh to two alternative products used for mesh fixation: Tisseel $^{\mathrm{TM}}$ fibrin sealant and the SorbaFix ${ }^{\mathrm{TM}}$ absorbable fixation system (tacks). For each of these products, the mesh displacement through the defect, the contact area, and the dislocation rate were monitored with applied pressure. Our results showed superior fixation of the ProGrip ${ }^{\text {TM }}$ laparoscopic self-fixating mesh compared to Tisseel ${ }^{\mathrm{TM}}$ fibrin sealant and to the SorbaFix ${ }^{\mathrm{TM}}$ tacks. Additionally, we found that mesh fixed with Tisseel ${ }^{\mathrm{TM}}$ fibrin sealant or SorbaFix ${ }^{\mathrm{TM}}$ tacks dislocated in some cases at intra-abdominal pressure. This observation suggests that in hernia repairs for which Tisseel $^{\text {TM }}$ fibrin sealant or SorbaFix ${ }^{\text {TM }}$ tacks are used, mesh dislocation could occur very early postoperatively, before the mesh has integrated into the tissue. While the dislocation of the ProGrip ${ }^{\mathrm{TM}}$ laparoscopic self-fixating mesh is also possible, no instances were observed in this ex vivo study, even at pressures well above those experienced in the abdomen.

Differences in mesh fixation could be attributed to the differences in the ways in which the fixation products are distributed. Tacks, such as SorbaFix ${ }^{\mathrm{TM}}$, only offer fixation at the tack sites, and might require some level of mesh incorporation for stability against intra-abdominal pressure. The angle of tack insertion has been shown to affect mesh fixation, ${ }^{30}$ and the number and spacing of tacks for optimal performance have not been determined. ProGrip ${ }^{\mathrm{TM}}$ laparoscopic self-fixating mesh provides homogenous fixation across the whole mesh surface. Because the polylactic acid microgrips are components of the mesh, they are likely to provide more consistent fixation.

All three of the fixation modalities that were tested have been used successfully in hernia repair procedures, but further studies are needed to determine which is optimal under specific circumstances. The clinical benefit of using absorbable tacks instead of nonabsorbable tacks has yet to be reported. Fibrin-based glue was first used experimentally for hernia mesh fixation over a decade $\mathrm{ago}^{31}$ and, since then, has quickly gained acceptance for use in clinical procedures. Fibrin glue has been shown to offer mesh fixation strength similar to other, more invasive fixation methods ${ }^{31,32}$ while causing less postoperative pain. ${ }^{33-35}$ Additionally, hernia recurrence rates in patients with fibrin glue fixation are no higher than in patients with traditional tack or suture fixation. ${ }^{34,36}$ Because the strength of fibrin glue varies significantly with different types of meshes, ${ }^{18,37}$ this method of fixation could potentially be used with incompatible products, which would render it less effective in those cases. Another characteristic of fibrin glues worth considering is their biodegradation. By covering the mesh with glue, integration of cells around the mesh fibers is delayed. It has been shown that degradation of fibrin glue is quite slow $^{38}$ and that complete degradation can take several months. ${ }^{39}$

While the results from this study clearly showed stronger fixation with the ProGrip ${ }^{\mathrm{TM}}$ laparoscopic self-fixating mesh compared to the other products, there are several caveats. Swine abdomens were used as a model for products typically used in inguinal hernia repair because there are currently no animal models for inguinal hernia. While results in this animal model still provide insight into the efficacy of mesh fixation to physiological tissue, clinical studies are needed to better assess these fixation modalities for their intended purpose. Previously, there was no available evidence to show whether self-fixating meshes pose an increased risk of early postoperative mesh dislocation. As a precaution to assure that the sample size for biomechanical testing would not be reduced because of mesh dislocation during the first 24 postoperative hours, four sutures were applied to secure the mesh in both the ProGrip ${ }^{\mathrm{TM}}$ laparoscopic self-fixating mesh group and the Bard $^{\mathrm{TM}}$ Soft Mesh + Tisseel ${ }^{\mathrm{TM}}$ group. However, a randomized, 
controlled trial was recently completed in which Parietex ProGrip ${ }^{\mathrm{TM}}$ was shown to be effective in open inguinal hernia repair and to be associated with a low recurrence rate when either zero or one stitch was used to facilitate mesh placement. ${ }^{40}$ The role of the peritoneum in mesh fixation and defect strengthening was not explored in the current study because the meshes were fixed to the rectus muscles only and not to the peritoneum. Future studies are needed to assess the effects of the peritoneum on the mechanical strength of mesh repair. The caveats described above do not impact the results of the study, but additional studies will surely provide further insight on the use of these fixation modalities.

The ProGrip ${ }^{\mathrm{TM}}$ laparoscopic self-fixating mesh was recently developed; it is based on the validated ProGrip ${ }^{\mathrm{TM}}$ technology, so it offers the benefits of tack-free fixation with the convenience of using only a single product. This might facilitate a more standardized procedure for hernia repair. Surgeon experience and skill are among the most prominent factors determining the success of an operation, and using only a single product decreases both operating time ${ }^{41}$ and opportunities for surgeon error. Finally, results from this study demonstrate that the ProGrip ${ }^{\mathrm{TM}}$ laparoscopic self-fixating mesh is superior to the Tisseel ${ }^{\mathrm{TM}}$ fibrin sealant and the SorbaFix $^{\mathrm{TM}}$ absorbable fixation system in its fixation strength and dislocation rate. While analysis 24 hours after surgery is sufficient for comparing mesh fixation alone, our study does not consider mesh integration or the long-term effects of article implantation with the different fixation modalities. Future studies on patients are needed to assess whether these results correlate with the clinical benefits so that surgeons are better able to provide the most effective product for each hernia patient. Additionally, many other factors beyond the strength of mesh fixation in the early postoperative period contribute to hernia recurrence, and all of these must be considered to maximize the chance of operative success.

\section{Conclusion}

Under the study conditions, the ProGrip ${ }^{\mathrm{TM}}$ laparoscopic self-fixating mesh exhibited superior fixation performance compared to the other tested fixation modalities. This result is to be confirmed by clinical data.

\section{Acknowledgments}

The authors would like to thank NAMSA for performing the surgical procedure and sample collection, and the Plateforme Biomecanique Experimentale of Laboratoire de Biomecanique des Chocs (Institut Français des Sciences et Technologies des Transports, de l'Aménagement et des
Réseaux; Université Claude Bernard Lyon 1) for access to its testing facilities. Medical writing and editing support was provided by Caroline Ritchie, $\mathrm{PhD}$ (Covidien).

\section{Disclosure}

This study was sponsored by Covidien, and Gaëtan Guérin, Xavier Bourges, and Frédéric Turquier are full-time employees of the company. The authors report no other conflicts of interest in this work.

\section{References}

1. EU Hernia Trialists Collaboration. Laparoscopic compared with open methods of groin hernia repair: systematic review of randomized controlled trials. Br J Surg. 2000;87(7):860-867.

2. Fränneby U, Sandblom G, Nordin P, Nyrén O, Gunnarsson U. Risk factors for long-term pain after hernia surgery. Ann Surg. 2006;244(2): 212-219.

3. EU Hernia Trialists Collaboration. Mesh compared with non-mesh methods of open groin hernia repair: systematic review of randomized controlled trials. Br J Surg. 2000;87(7):854-859.

4. EU Hernia Trialists Collaboration. Repair of groin hernia with synthetic mesh: meta-analysis of randomized controlled trials. Ann Surg. 2002;235(3):322-332.

5. Byrd JF, Agee N, Swan RZ, et al. Evaluation of absorbable and permanent mesh fixation devices: adhesion formation and mechanical strength. Hernia. 2011;15(5):553-558.

6. Bansal VK, Misra MC, Babu D, et al. A prospective, randomized comparison of long-term outcomes: chronic groin pain and quality of life following totally extraperitoneal (TEP) and transabdominal preperitoneal (TAPP) laparoscopic inguinal hernia repair. Surg Endosc. 2013;27(7):2373-2382.

7. Tolver MA, Rosenberg J, Bisgaard T. Early pain after laparoscopic inguinal hernia repair. A qualitative systematic review. Acta Anaesthesiol Scand. 2012;56(5):549-557.

8. Ismail M, Garg P. Laparoscopic inguinal total extraperitoneal hernia repair under spinal anesthesia without mesh fixation in 1,220 hernia repairs. Hernia. 2009;13(2):115-119.

9. Fortelny RH, Petter-Puchner AH, Glaser KS, Redl H. Use of fibrin sealant (Tisseel/Tissucol) in hernia repair: a systematic review. Surg Endosc. 2012;26(7):1803-1812.

10. Sajid MS, Ladwa N, Kalra L, McFall M, Baig MK, Sains P. A meta-analysis examining the use of tacker mesh fixation versus glue mesh fixation in laparoscopic inguinal hernia repair. Am J Surg. 2013;206(1):103-111.

11. Schwab R, Schumacher O, Junge K, Binnebösel M, Klinge U, Schumpelick V. Fibrin sealant for mesh fixation in Lichtenstein repair: biomechanical analysis of different techniques. Hernia. 2007;11(2): 139-145.

12. Kingsnorth A, Gingell-Littlejohn M, Nienhuijs S, et al. Randomized controlled multicenter international clinical trial of self-gripping Parietex ${ }^{\mathrm{TM}}$ ProGrip ${ }^{\mathrm{TM}}$ polyester mesh versus lightweight polypropylene mesh in open inguinal hernia repair: interim results at 3 months. Hernia. 2012;16(3):287-294.

13. Duffy AJ, Hogle NJ, LaPerle KM, Fowler DL. Comparison of two composite meshes using two fixation devices in a porcine laparoscopic ventral hernia repair model. Hernia. 2004;8(4):358-364.

14. Krpata DM, Blatnik JA, Harth KC, Phillips MS, Novitsky YW, Rosen MJ. Evaluation of fibrin sealant for biologic mesh fixation at the hiatus in a porcine model. Surg Endosc. 2012;26(11):3120-3126.

15. McGinty JJ, Hogle NJ, McCarthy H, Fowler DL. A comparative study of adhesion formation and abdominal wall ingrowth after laparoscopic ventral hernia repair in a porcine model using multiple types of mesh. Surgi Endosc. 2005;19(6):786-790. 
16. Fortelny RH, Petter-Puchner AH, Ferguson J, et al. A comparative biomechanical evaluation of hernia mesh fixation by fibrin sealant. J Surg Res. 2011;171(2):576-581.

17. Melman L, Jenkins ED, Deeken CR, et al. Evaluation of acute fixation strength for mechanical tacking devices and fibrin sealant versus polypropylene suture for laparoscopic ventral hernia repair. Surg Innov. 2010;17(4):285-290.

18. Rieder E, Stoiber M, Scheikl V, et al. Mesh fixation in laparoscopic incisional hernia repair: glue fixation provides attachment strength similar to absorbable tacks but differs substantially in different meshes. J Am Coll Surg. 2011;212(1):80-86.

19. Schug-Pass C, Lippert H, Köckerling F. Fixation of mesh to the peritoneum using a fibrin glue: investigations with a biomechanical model and an experimental laparoscopic porcine model. Surg Endosc. 2009;23(12):2809-2815.

20. Fortuny G, Rodríguez-Navarro J, Susín A, López-Cano M. Simulation and study of the behaviour of the transversalis fascia in protecting against the genesis of inguinal hernias. J Biomech. 2009;42(14):2263-2267.

21. Guérin G, Turquier F. Impact of the defect size, the mesh overlap and the fixation depth on ventral hernia repairs: a combined experimental and numerical approach. Hernia. 2013;17(5):647-655.

22. Hernández-Gascón B, Grasa J, Peña E, Pascual G, Bellón JM, Calvo B. Numerical modelling of the abdominal wall applied to hernia surgery. J Biomech. 2012;45:S567.

23. Lubowiecka I, Szymczak C, Tomaszewska A, Śmietański M. Mathematical study of a tissue-implant connection in a ventral hernia repair in a context of the system's parameters. Proc Appl Math Mech. 2009;9(1):167-168.

24. Binnebösel M, Rosch R, Junge K, et al. Biomechanical analyses of overlap and mesh dislocation in an incisional hernia model in vitro. Surgery. 2007;142(3):365-371.

25. Podwojewski F, Otténio M, Beillas P, Guérin G, Turquier F, Mitton D. Mechanical response of animal abdominal walls in vitro: evaluation of the influence of a hernia defect and a repair with a mesh implanted intraperitoneally. J Biomech. 2013;46(3):561-566.

26. Schwab R, Schumacher O, Junge K, et al. Biomechanical analyses of mesh fixation in TAPP and TEP hernia repair. Surg Endosc. 2008;22(3):731-738.

27. Cobb WS, Burns JM, Kercher KW, Matthews BD, James Norton H, Todd Heniford B. Normal intraabdominal pressure in healthy adults. J Surg Res. 2005;129(2):231-235.

28. Bellows CF, Smith A, Malsbury J, Helton WS. Repair of incisional hernias with biological prosthesis: a systematic review of current evidence. Am J Surg. 2013;205(1):85-101.
29. Slater NJ, van der Kolk M, Hendriks T, van Goor H, Bleichrodt RP. Biologic grafts for ventral hernia repair: a systematic review. Am J Surg. 2013;205(2):220-230.

30. Sadava EE, Krpata DM, Gao Y, Schomisch S, Rosen MJ, Novitsky YW. Laparoscopic mechanical fixation devices: does firing angle matter? Surg Endosc. 2013;27(6):2076-2081.

31. Katkhouda N, Mavor E, Friedlander MH, et al. Use of fibrin sealant for prosthetic mesh fixation in laparoscopic extraperitoneal inguinal hernia repair. Ann Surg. 2001;233(1):18-25.

32. Katkhouda N. A new technique for laparoscopic hernia repair using fibrin sealant. Surg Technol Int. 2004;12:120-126.

33. Helvind NM, Andresen K, Rosenberg J. Lower reoperation rates with the use of fibrin sealant versus tacks for mesh fixation. Surg Endosc. 2013;27(11):4184-4191.

34. Tolver MA, Rosenberg J, Juul P, Bisgaard T. Randomized clinical trial of fibrin glue versus tacked fixation in laparoscopic groin hernia repair. Surg Endosc. 2013;27(8):2727-2733.

35. Campanelli G, Pascual MH, Hoeferlin A, et al. Randomized, controlled, blinded trial of Tisseel/Tissucol for mesh fixation in patients undergoing Lichtenstein technique for primary inguinal hernia repair: results of the TIMELI trial. Ann Surg. 2012;255(4):650-657.

36. Kaul A, Hutfless S, Le H, et al. Staple versus fibrin glue fixation in laparoscopic total extraperitoneal repair of inguinal hernia: a systematic review and meta-analysis. Surg Endosc. 2012;26(5):1269-1278.

37. Schug-Pass C, Jacob DA, Lippert H, Köckerling F. Differences in biomechanical stability using various fibrin glue compositions for mesh fixation in endoscopic inguinal hernia repair. Surg Endosc. 2012;26(11): 3282-3286.

38. Silverman RP, Passaretti D, Huang W, Randolph MA, Yaremchuk MJ. Injectable tissue-engineered cartilage using a fibrin glue polymer. Plast Reconstr Surg. 1999;103(7):1809-1818.

39. Kroez M, Lang W, Dickneite G. Wound healing and degradation of the fibrin sealant Beriplast $\mathrm{P}$ following partial liver resection in rabbits. Wound Repair Regen. 2005;13(3):318-323.

40. Sanders DL, Nienhuijs S, Ziprin P, Miserez M, Gingell-Littlejohn M, Smeds S. Randomized clinical trial comparing self-gripping mesh with suture fixation of lightweight polypropylene mesh in open inguinal hernia repair. Br J Surg. 2014;101(11):1373-1382.

41. Cambal M, Zonca P, Hrbaty B. Comparison of self-gripping mesh with mesh fixation with fibrin-glue in laparoscopic hernia repair (TAPP). Bratisl Lek Listy. 2012;113(2):103-107.
Medical Devices: Evidence and Research

\section{Publish your work in this journal}

Medical Devices: Evidence and Research is an international, peerreviewed, open access journal that focuses on the evidence, technology, research, and expert opinion supporting the use and application of medical devices in the diagnosis, treatment and management of clinical conditions and physiological processes. The identification of novel

\section{Dovepress}

devices and optimal use of existing devices which will lead to improved clinical outcomes and more effective patient management and safety is a key feature. The manuscript management system is completely online and includes a quick and fair peer-review system. Visit http://www. dovepress.com/testimonials.php to read real quotes from authors. 